was the average, 97.6 and $107 \mathrm{~F}$. representing the extremes.

A pseudo-crisis occurred and there was none of the usual causes to account for the return of fever. There was no new lung involvement nor abscess formation.

Although there was some stupor at first, the meningeal symptoms were slight until after the pseudo-crisis. Then symptoms developed very suggestive of a meningitis, except that they were of a changeable nature. The fact that recovery ensued shows that Cheyne-Stokes respiration is not always significant of a fatal termination.

Treatment in these cases is clear. The main attention should be paid to careful feeding and free elimination. I have found that by using rather large doses of sodium sulphocarbolate and small, frequent doses of aromatic fluid extract of cascara the intestinal condition can be markedly improved. In only the more asthenic cases has there been an urgent demand for calomel and colonic irrigation.

I have also noticed marked improvement following the nee of a diuretic.

Potassium citrate, at times theobromin-sodium salicylate, and normal salt solution I consider the best. The latter is best given in enemas.

\section{SPONTANEOUS ARTERIOSCLEROSIS OF THE AORTA (ATHEROMA) IN A RABBIT.*}

W. OPHÜLS, M.D.

Professor of Pathology and Bacteriology Cooper Medical College. SAN FRANCISCO.

So much has been written lately about the artificial production of aortic lesions in rabbits by intravenous injections of adrenalin, nicotin and other substances, and their relation to the lesions in human arterial dis-

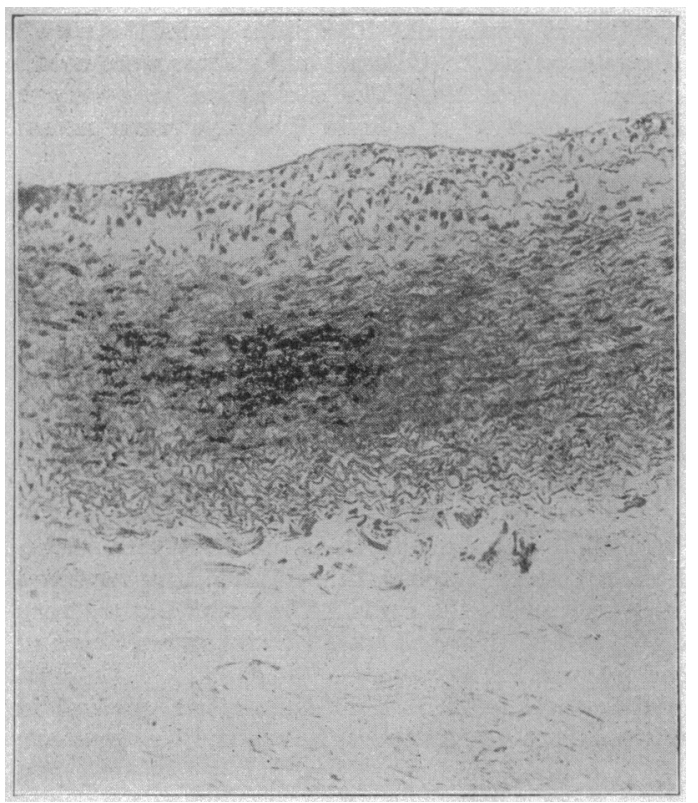

Figure 1.-Photomicrograph of section of the diseased aorta showing histologic character of lesion (Hematoxylin-eosin).

ease, that it would seem of interest to record a case of spontaneous atheroma accidentally observed in a rabbit.

* From the Pathological Laboratory of Cooper Medical College, San Francisco, Cal.

The rabbit in question was a large, healthy, welldeveloped female, raised in the country, which had just been received by the laboratory and had not been at the college over eight days. It died of acute anemia in the course of an unsuccessful surgical operation.

Examination of the aorta showed a normal caliber and normal elasticity of the wall. In the arch near its beginning there were several light yellow, fairly well circumscribed, slightly raised, oblonf spots from 2 to $3 \mathrm{~mm}$. in greatest diameter, which attracted attention on ac-

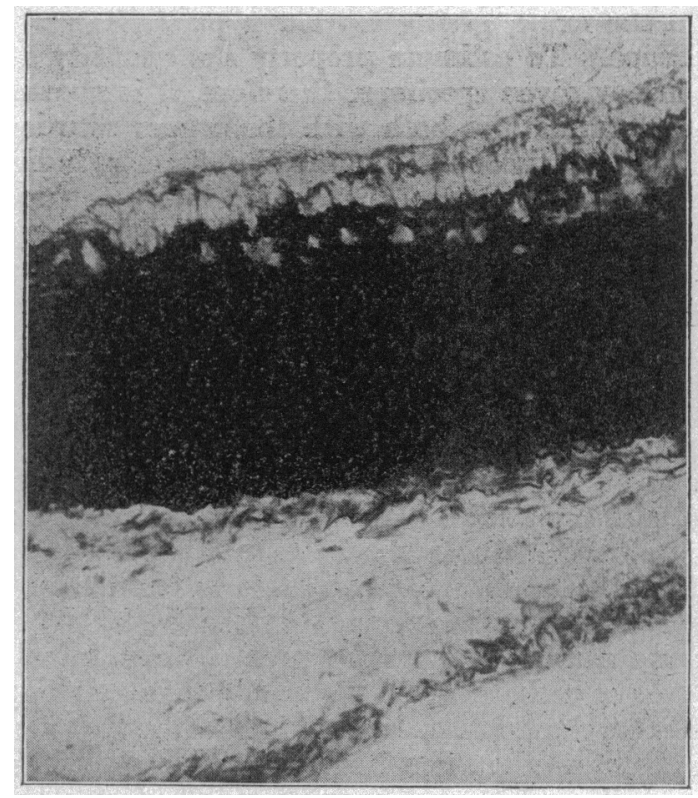

Figure 2.-Photomicrograph of a section of the diseased aorta stained with Wlegert's stain for elastic fibers, showing separation of elastic membranes.

count of their great similarity to the initial stages of atheroma in man and their dissimilarity to the lesions produced by adrenalin injections.

Sections confirmed this impression. As shown in the photomicrograph, the lesion is situated very largely on the inside of the blood vessel in the region of the intima. The upper layers of the muscle are also involved and the elastic membranes separated more or less from one another, as is shown in the other photomicrograph. This early involvement of the muscle is not astonishing when we remember that in rabbits the intima practically consists of the endothelial layer only. Histologically the lesion is an exact counterpart of the early lesions in human atheroma which I had much opportunity to study in my recent work on human arteriosclerosis. ${ }^{1}$ We have the same accumulation of large cells (probably proliferated connective tissue cells) in the tissues with marked fatty degeneration of their protoplasm. In the rabbit also the trouble is not confined to the intima and the upper layers of the muscle, but the adventitia also seems to be involved. The sections show a moderate, but quite distinct fibrous thickening of the latter underneath the plaques, and in serial sections I even discovered an area of cellular infiltration around one of the vasa vasorum in the thickened adventitia.

So far as this observation goes the spontaneous atheroma in rabbits would appear to be much more like that of man than the experimental lesions produced with adrenalin.

Arteriosclerosis of the Aorta, Am. Jour. of Med. Scl., June 906. 